\title{
Positive streamers in short and large gaps in hydrocarbons of various chemical natures
}

\author{
O. Lesaint and A. Denat \\ Univ. Grenoble Alpes, G2Elab, F-38000 Grenoble, France \\ CNRS, G2Elab, F-38000 Grenoble, France
}

\author{
F. Mc Cluskey \\ Univ. Grenoble Alpes, LEGI, F-38000 Grenoble, France \\ CNRS, LEGI, F-38000 Grenoble, France
}

\begin{abstract}
The objective of this study is to document the relationship existing between liquid composition and streamer propagation in large gaps. Measurements are carried out in a series of hydrocarbon liquids of various chemical natures (aliphatic, aromatic, polyaromatic), both in short point-plane gap (6 $\mathrm{mm}$ distance, $1 \mu \mathrm{m}$ tip radius), and in larger gaps up to $20 \mathrm{~cm}$. These measurements are based on fast imaging techniques (2D and streak photographs), coupled to transient current and light recording. A correlation is established between the threshold propagation voltage of filamentary streamers, the tendency to form branches, and the behaviour at very high voltage.
\end{abstract}

Keywords-Streamer; insulating liquids; propagation velocity; breakdown voltage; acceleration voltage

\section{INTRODUCTION}

Studies of prebreakdown phenomena ("streamers") carried out at large gaps and very high voltage in the past decades have evidenced the existence of several "propagation modes" [1]. When voltage is increased, streamer propagation velocity shows sudden transitions to higher values, accompanied with marked changes in streamer shapes, currents, etc. Although physical processes involved during propagation are still not totally clear today, these propagation "modes" constitutes a convenient way to clarify the description of events.

In point-plane gaps, the positive " 2 nd mode" in mineral transformer oil is responsible for breakdown over a large range of distances and voltage, and it shows a great stability. With a $10 \mathrm{~cm}$ gap distance, increasing the voltage by a factor a 5 (from 70 to $350 \mathrm{kV}$ ) induces only a small increase of velocity, from 1.5 to $3 \mathrm{~km} / \mathrm{s}$ [1]. In order to observe faster streamer modes (with average velocity up to $100 \mathrm{~km} / \mathrm{s}$ ) in mineral oil, it is necessary to apply very high voltage, far exceeding the breakdown voltage. This behaviour was attributed to a very efficient "self-stabilization" mechanism in this liquid, presumably due to a marked tendency to form new streamer branches when voltage is increased [1]. This hypothesis was supported by experiments carried out with tubes [2], and also by results obtained with low ionization potential additives (pyrene in cyclohexane [3], and DMA in white oil [4]).

In small gap at very low voltage, close to the threshold propagation voltage $V_{p}$ of $2^{\text {nd }}$ mode streamers, the addition of pyrene to cyclohexane produces a decrease of $V_{p}$ (i.e. propagation is facilitated), correlated to a marked tendency to form new branches when voltage is increased [3]. In a large gap, the addition of pyrene leads to a behaviour identical to mineral oil: a large overvoltage is necessary to observe fast streamers. Without pyrene, positive streamers in pure cyclohexane are much more "unstable": they accelerate at much lower voltage (close to the breakdown voltage). A very similar unstable behaviour was also observed in esters $[5,6]$. These observations are consistent with the presence of low ionization potential molecules in mineral oil (such as aromatics).

This study was carried out in order to further document the relationship existing between liquid composition and streamer propagation, by doing measurements in a series of pure hydrocarbon liquids of various chemical natures (aliphatic, aromatic, polyaromatic). As in [3], measurements are carried out in short point-plane gap (6 $\mathrm{mm}$ distance, $1 \mu \mathrm{m}$ tip radius) in order to measure $V_{p}$ and observe differences between streamer shapes (i.e. branching tendency). Measurements in larger gaps up to $20 \mathrm{~cm}$ allow us to study the influence of liquid composition on breakdown and onset of fast streamers.

\section{EXPERIMENTAL SYSTEMS}

\section{A. $6 \mathrm{~mm}$ point-plane gap and low voltage}

The experimental setup used was identical to the one described in [3]. A rectangular high voltage pulse was used to study streamer initiation and propagation. Its main feature is the fast rise time (about $20 \mathrm{~ns}$ ), in order to minimize the influence of injected space charges during the voltage rise, and take correct measurements of initiation voltage and threshold propagation voltage $V_{p}$. The electrode geometry was a pointplane gap with a fixed gap distance $(d=6 \mathrm{~mm})$ and a sharp point (tip radius of curvature $r_{p}=1 \mu \mathrm{m}$ ), necessary to measure properly $V_{p}$. Points were built with electrochemically etched tungsten wires. The plane electrode, $45 \mathrm{~mm}$ in diameter, was covered with a PTFE sheet (2 $\mathrm{mm}$ in thickness) in order to prevent the total breakdown (arc) from occurring.

The initiation of streamers was detected by high speed shadowgraphic visualisation with a high magnification. The optical resolution was about $1 \mu \mathrm{m}$. This was necessary since smallest streamers at the initiation voltage can be as short as a few $\mu \mathrm{m}$.

\section{B. Large gaps up to $20 \mathrm{~cm}$ and high voltage}

Experiments in larger gaps were carried out using the system described in [1] (figure 1). The high voltage impulse had a 0.4/1400 $\mu$ s shape and was delivered by a Marx generator. Photographs of the light emitted by streamers were obtained with a gated image intensifier (integral images) or with a streak camera. Light emissions from streamers were recorded with a photomultiplier, and streamer current (or charge) with a probe optically coupled to a fast oscilloscope. 
The test cell, 13 litres in volume, had a grounded plane electrode $20 \mathrm{~cm}$ in diameter, and gap distance $d$ up to $20 \mathrm{~cm}$. No insulating solid was placed on the plane. High voltage electrodes were steel points, $40 \mu \mathrm{m}$ in tip radius. A $320 \mathrm{ohm}$ resistor was placed in series with the high voltage connection. This resistor, together with the small energy available from the Marx generator (less than 400 Joules), contributed to limit the breakdown energy, and thus the degradation of the liquid and electrodes by successive breakdowns. types:

Hydrocarbon liquids investigated here are of different

- $\quad$ aromatic: monobenzyltoluene (BT), phenylxylylethane (PXE);

- polyaromatic: diisopropylnaphtalene (IPN);

- $\quad$ saturated: polybutene (PB);

- mineral oil (MO).

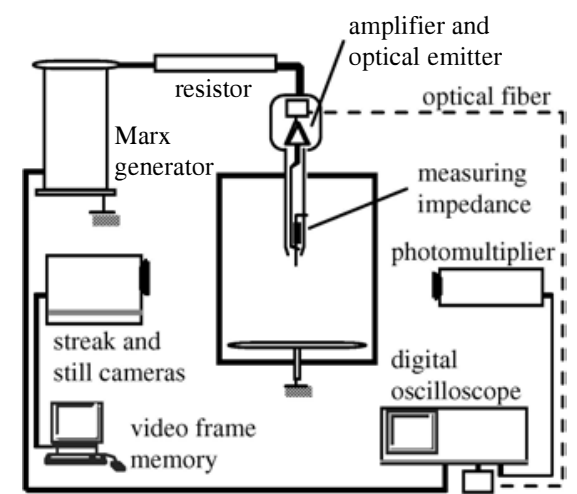

Fig. 1 Experimental setup used for large gap measurements [6]

\section{RESULTS AND DISCUSSIONS}

\section{A. Small gap experiments}

In all liquids, prebreakdown streamers observed at the lowest voltage are of " 1 st mode" (figure 2). Their main characteristics are a rounded shape, and a low propagation velocity, from about $10 \mathrm{~m} / \mathrm{s}$ up to some $100 \mathrm{~m} / \mathrm{s}$. Above some critical threshold voltage $V_{p}$, the streamer shape changes to a filamentary shape with a higher velocity, and this process is correlated to the sudden increase of the transient current [3].

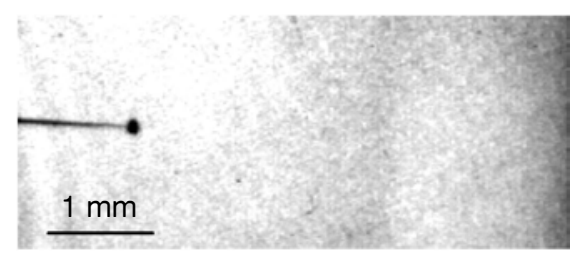

Fig. 2 " 1 st mode" slow positive streamer in BT $(V=10 \mathrm{kV})$.

Figure 3 shows the percentage of filamentary streamers versus voltage. To obtain this percentage, twenty shots were applied at each voltage level. The threshold propagation voltage $V_{p}$ corresponds to a $50 \%$ probability. On figure 3 , we have also added for comparison measurements carried out in identical conditions in pure cyclohexane $(\mathrm{CH})$, and in cyclohexane + pyrene (CH + PY, 1 mol/liter concentration) [3].

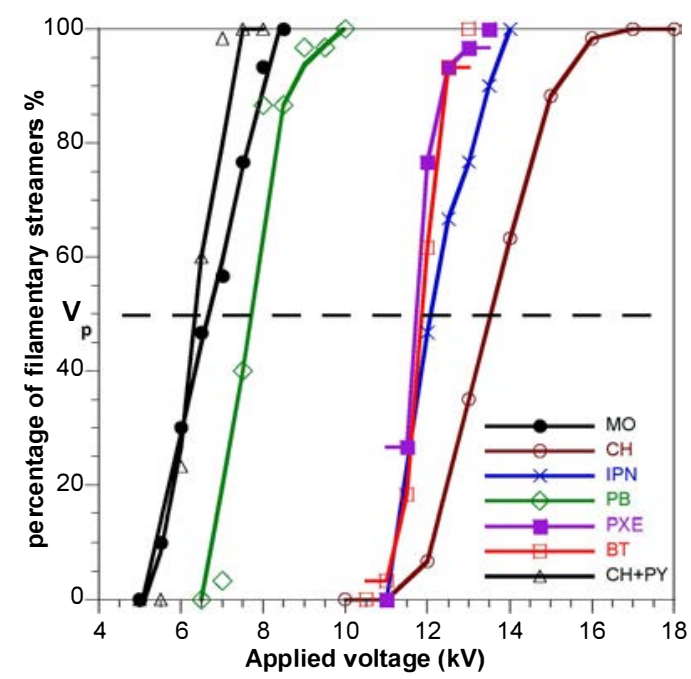

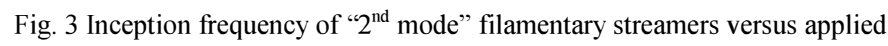
voltage

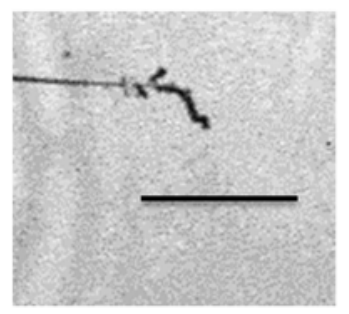

$\mathrm{a}-\mathrm{PB} 10 \mathrm{kV}$

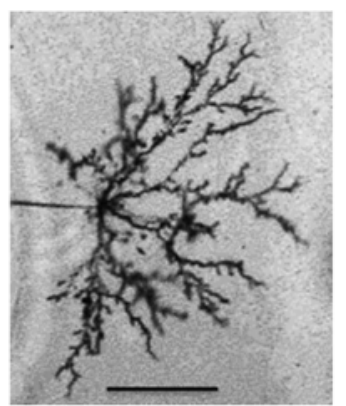

c - PB $25 \mathrm{kV}$

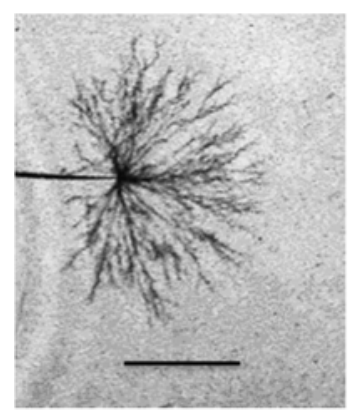

e - MO $30 \mathrm{kV}$

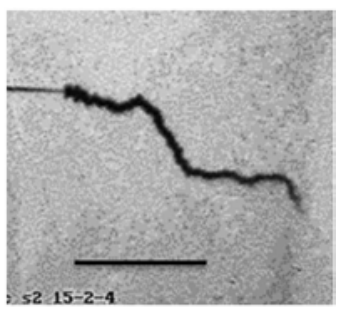

b - BT $15 \mathrm{kV}$

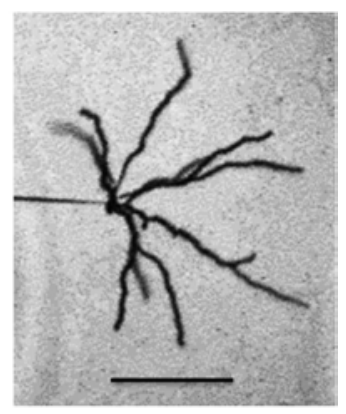

d - BT $20 \mathrm{kV}$

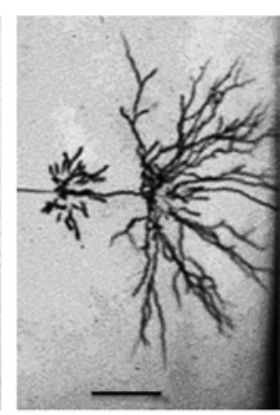

f - BT $30 \mathrm{kV}$
Fig. 4 Typical shapes of $2^{\text {nd }}$ mode positive streamers. Black line: $1 \mathrm{~mm}$ scale. 
According to the liquid nature, two main groups can be observed on figure 3 :

- Group I: MO, PB, and CH + PY, characterized by a low value of $V_{p}$ (from 6 to $7.5 \mathrm{kV}$ );

- Group II: PXE, BT, IPN and $\mathrm{CH}$, characterized by a much higher value of $V_{p}$ (from 11.5 to $13.5 \mathrm{kV}$ ).

The aromatic and polyaromatic PXE, BT, and IPN show a nearly identical behaviour.

Within the investigated voltage range from $V_{p}$ to $30 \mathrm{kV}$, propagation velocities of $2^{\text {nd }}$ mode filamentary streamers in all liquids are in the range of $0.5-2 \mathrm{~km} / \mathrm{s}$. Streamer shapes show large variations according to the liquid nature (Fig. 4).

Close to the propagation threshold $V_{p}$, streamers in all liquids are frequently composed by a single main filament (a, b). However, in $\mathrm{MO}$ and $\mathrm{PB}$, some small side-branches are already seen (a), whereas in BT the filament shows no branching during propagation (b). These differences become much more marked at higher voltage (c and d). A considerable number of small side-branches appear in PB, whereas filaments initiated at the point in BT show almost no branching.

At $30 \mathrm{kV}$, streamers in liquids of group I (MO on Fig. 4e) show a very large number of filaments with many sidebranches, contained within a nearly spherical envelope. Liquids of group II show either few filaments (in $\mathrm{CH}$ [3]), or the unusual shape seen in aromatics (BT on Fig. 4f, previously described in [7]). On this figure, a new secondary structure appears during propagation at the extremity of one filament.

\section{B. Large gap experiments}

Prebreakdown and breakdown measurements were carried out in large point-plane gaps up to $20 \mathrm{~cm}$ with a step voltage $(0.4 / 1400 \mu \mathrm{s})$ in MO, PB, IPN, BT and PXE. Breakdown voltages $V_{b}$ (corresponding to a $50 \%$ probability) versus gap distance $d$ are shown in figure 5. Different trends are observed between liquids. With liquids of group I (Mineral oil and polybutene $\mathrm{PB}$ ), a nearly linear increase of $V_{b}$ versus $d$ is observed, with an average slope of $11.9 \mathrm{kV} / \mathrm{cm}$. In BT and PXE, an increase is also observed, but with a lower average slope $(7 \mathrm{kV} / \mathrm{cm}$, between 5 and $20 \mathrm{~cm})$. In IPN, an intermediate trend is observed, with a more curved plot, which slope decreases with distance.

Figure 6 shows the "breakdown velocity" $v_{b}$ calculated by dividing the distance $d$ by the time to breakdown $t_{b}$ measured during breakdown experiments. Here again, marked differences are seen between liquids. Liquids of group I (mineral oil and polybutene) show a great stability, with a velocity almost unchanged over the investigated range, and close to values measured in short gap $(1-2 \mathrm{~km} / \mathrm{s})$.

In aromatic liquids (BT and PXE), $v_{b}$ is much higher (from 6 to $24 \mathrm{~km} / \mathrm{s}$ in BT) and increases with voltage. It is not any more comparable to values measured in short gap. This means that streamers in these liquids already accelerated at voltages below the breakdown voltage, whereas acceleration in mineral oil requires a large overvoltage, about twice the breakdown voltage [1].

The polyaromatic liquid IPN shows an intermediate behaviour, with acceleration observed at distances larger than
$15 \mathrm{~cm}$. At $d=20 \mathrm{~cm}$, IPN shows a breakdown voltage identical to mineral oil and polybutene. However, streamers already accelerated in IPN whereas in MO and PB the velocity remains stable, even when the voltage is raised above $V_{b}$.

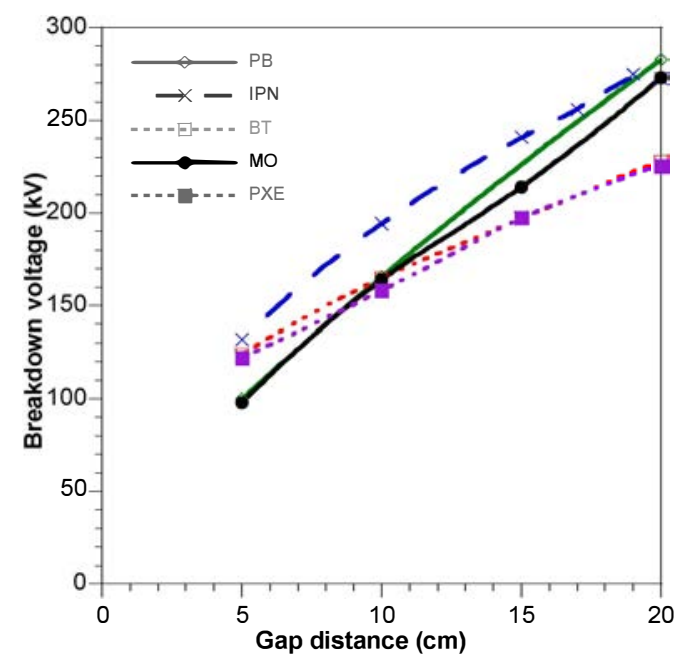

Fig. 5 Breakdown voltage ( $50 \%$ probability) versus gap distance with a $0.4 / 1400 \mu$ s wave.

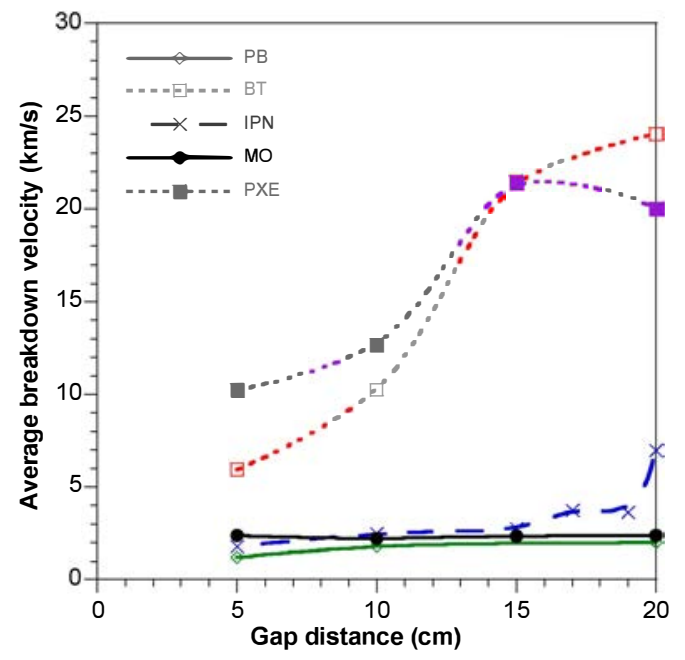

Fig. 6 Average breakdown velocity versus gap distance with a $0.4 / 1400 \mu$ s wave.

\section{Fast streamers in aromatic BT}

Figure 7 shows the average streamer propagation velocity in BT, measured in $20 \mathrm{~cm}$ gap below the breakdown voltage. The average streamer velocity was calculated from the streamer propagation length, and from the duration of current and light signals. These measurements show that streamers in BT indeed already accelerated at voltage above $180 \mathrm{kV}$, i.e. below the breakdown voltage. A very steep acceleration (up to $100 \mathrm{~km} / \mathrm{s}$ ) is seen at $240 \mathrm{kV}$. Similar measurements in IPN indicate a steep acceleration at $280 \mathrm{kV}$, whereas in polybutene the velocity remained at $2 \mathrm{~km} / \mathrm{s}$ at $300 \mathrm{kV}$.

Figure 8 shows a typical recording of transient current, light emission, and streak image of a fast streamer propagating in BT. The propagation occurs in fast discrete steps. During each step, a current pulse is recorded while the very luminous 
streamer head propagates, starting from the extremity of the channel created at the previous step. Periods of inactivity are recorded between steps. Correlating the length of steps to the duration of the corresponding current pulses indicates an instantaneous velocity of $140 \mathrm{~km} / \mathrm{s}$ during steps, whereas the average velocity (calculated by including periods of inactivity) is $20 \mathrm{~km} / \mathrm{s}$ on this example. These features are nearly identical to those observed in esters $[5,6]$.

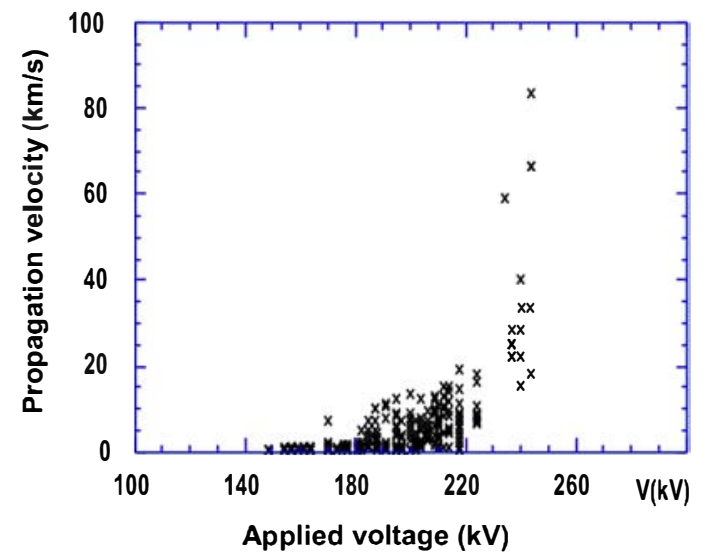

Fig. 7 Streamer propagation velocity versus voltage, measured in BT in a $20 \mathrm{~cm}$ gap below the breakdown voltage $(0.4 / 1400 \mu$ s wave $)$.

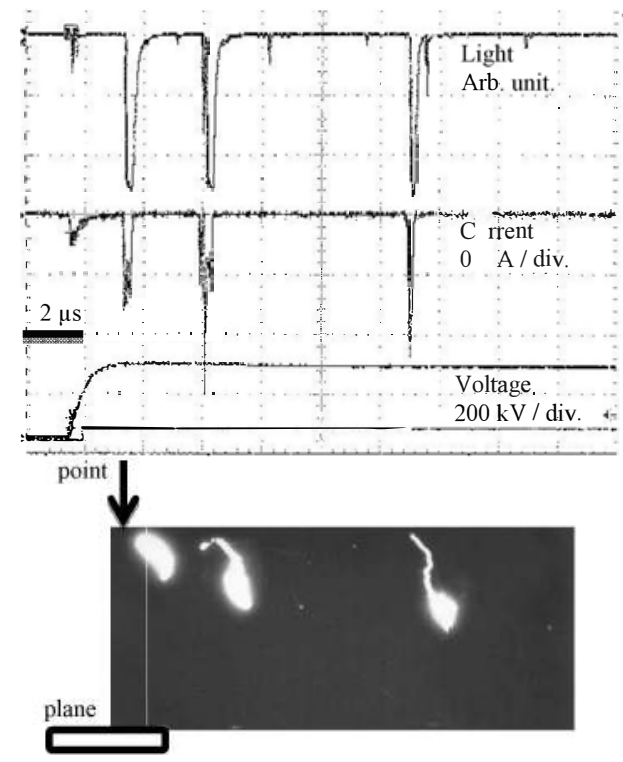

Fig. 8 Light, current and streak photograph recorded during the propagation of a fast streamer in BT. Light and current appear as negative signals. The horizontal time scale on oscilloscope recording and streak photograph are identical. ( $20 \mathrm{~cm}$ gap, $215 \mathrm{kV}, 0.4 / 1400 \mu \mathrm{s}$ wave).

\section{CONCLUSIONS}

Pre-breakdown and breakdown measurements carried out in a variety of hydrocarbon liquids over a wide range of gap distances and applied voltage (from the minimum propagation voltage, up to very high voltage), confirm the conclusions previously obtained in cyclohexane with pyrene as additive [3]. Liquids characterized by a low threshold propagation voltage $V_{p}$ (mineral oil, polybutene, cyclohexane+pyrene) show a great stability of velocity at large gaps and high voltage. As in mineral oil, breakdown in large gaps is due to $2^{\text {nd }}$ mode streamers with a moderate velocity about $1-2 \mathrm{~km} / \mathrm{s}$. A large overvoltage is necessary to induce fast streamers in these liquids. Other measurements carried out in polybutene with lightning impulses showed that this liquid behaves in a very similar way as mineral oil, with a nearly identical value of the "acceleration voltage", well above the breakdown voltage.

Liquids characterized by a high propagation threshold voltage $V_{p}$ (PXE, BT, $\left.\mathrm{CH}, \mathrm{IPN}\right)$ show a much lower stability, such as previously observed in esters or in cyclohexane. In these liquids, streamers switch to fast propagation modes at lower voltage: below the breakdown voltage $V_{b}$ in PXE and $\mathrm{BT}$, and close to $V_{b}$ in IPN. Since fast streamers channels are also characterized by a higher conductivity [2], the increase of breakdown voltage versus gap distance is also slower compared to $2^{\text {nd }}$ mode streamers (figure 5). Fast streamers thus combine the disadvantages of high velocity (unfavourable for the performance of insulation under fast transients such as lightning impulse), and lower breakdown voltage in large gaps.

The shapes of streamers observed in small gaps confirm that the "branching tendency" is of great importance for the propagation under high voltage.

\section{ACKNOWLEDGMENTS}

The authors would like to acknowledge Mr P. Jay and Mrs N. Berger, from the former ELF Atochem / Arkema company, for their help and support for this study. Some of the experiments presented here were carried out by Mr F. Roldan, who is also acknowledged.

\section{REFERENCES}

[1] O. Lesaint and G. Massala, "Positive streamer propagation in large oil gaps: experimental characterization of propagation modes", IEEE Trans. Dielectr. Electr. Insul., Vol.5, No.3, pp.360-370, 1998.

[2] G. Massala and O. Lesaint, "Positive streamer propagation in large oil gaps. Electrical properties of streamers", IEEE Trans. on Dielectrics and Electrical Insulation, Vol. 5, n³, pp. 371-381, 1998.

[3] O. Lesaint and M. Jung, "On the relationship between streamer branching and propagation in liquids: influence of pyrene in cyclohexane.", J. Phys. D: Appl. Phys, 33, pp. 1360-1368, 2000.

[4] N.V. Dung, H.K. Hoidalen, D. Linhjell, L.E. Lundgaard, M.D. Unge, "Influence of impurities and additives on positive streamers in paraffinic model oil", IEEE Trans. Dielectr. Electr. Insul., Vol.19, No.5, pp.1593 1603,2012

[5] C. Tran Duy, O. Lesaint, A. Denat, N. Bonifaci, "Streamer Propagation and Breakdown in Natural Ester at High Voltage", IEEE Trans on Diel. And Elec. Insul., Vol. 16, Issue 6, pp. 1582-1594, 2009.

[6] Q. Liu and Z.D. Wang, "Streamer characteristic and breakdown in synthetic and natural ester transformer liquids under standard lightning impulse voltage", IEEE Trans. Dielectr. Electr. Insul., vol. 18, pp. 285294, 2011.

[7] O. Lesaint and F. Roldan, "On the correlation between positive streamer shape and propagation in benzyltoluene", 13th. Int. Conf. on Cond. and Break. in Diel. Liq. (ICDL), IEEE, pp. 167-170, Nara (Japan) July20-25, 1999 